Research Article

\title{
Extracts of Perilla frutescens var. Acuta (Odash.) Kudo Leaves Have Antitumor Effects on Breast Cancer Cells by Suppressing YAP Activity
}

\author{
Cho-Long Kim, ${ }^{1}$ Yu-Su Shin, ${ }^{2}$ Sue-Hee Choi, ${ }^{1}$ Seroc Oh, ${ }^{1}$ Kyeongseob Kim, \\ Han-Sol Jeong $\left(\mathbb{C}^{3}\right.$, and Jung-Soon Mo $\mathbb{\circledR}^{1,4}$ \\ ${ }^{1}$ Department of Biomedical Sciences, Graduate School, Ajou University School of Medicine, Suwon 16499, Republic of Korea \\ ${ }^{2}$ Department of Ginseng and Medicinal Herb, National Institute of Horticultural and Herbal Science (NIHHS), \\ Rural Development Administration (RDA), Wanju, Jeollabuk-Do 55365, Republic of Korea \\ ${ }^{3}$ Division of Applied Medicine, School of Korean Medicine, Pusan National University, Yangsan 50612, Republic of Korea \\ ${ }^{4}$ Institute of Medical Science, Ajou University School of Medicine, Suwon 16499, Republic of Korea
}

Correspondence should be addressed to Han-Sol Jeong; jhsol33@pusan.ac.kr and Jung-Soon Mo; j5mo@ajou.ac.kr

Received 10 February 2020; Revised 7 December 2020; Accepted 15 January 2021; Published 15 February 2021

Academic Editor: Elia Ranzato

Copyright $\odot 2021$ Cho-Long Kim et al. This is an open access article distributed under the Creative Commons Attribution License, which permits unrestricted use, distribution, and reproduction in any medium, provided the original work is properly cited.

Yes-associated protein (YAP)/WW domain-containing transcription factor (TAZ) is critical for cell proliferation, survival, and self-renewal. It has been shown to play a crucial oncogenic role in many different types of tumors. In this study, we investigated the antitumor effect of the extracts of Perilla frutescens var. acuta (Odash.) Kudo leaves (PLE) on Hippo-YAP/TAZ signaling. PLE induced the phosphorylation of YAP/TAZ, thereby inhibiting their activity. In addition, the treatment suppresses YAP/ TAZ transcriptional activity via the dissociation of the YAP/TAZ-TEAD complex. To elucidate the molecular mechanism of PLE in the regulation of YAP activity, we treated WT and cell lines with gene knockout (KO) for Hippo pathway components with PLE. The inhibitory effects of PLE on YAP-TEAD target genes were significantly attenuated in LATS1/2 KO cells. Moreover, we found the antitumor effect of PLE on MDA-MB-231 and BT549, both of which are triple-negative breast cancer (TNBC) cell lines. PLE reduced the viability of TNBC cells in a dose-dependent manner and induced cell apoptosis. Further, PLE inhibited the migration ability in MDA-MB-231 cells. This ability was weakened in YAP and TEAD-activated clones suggesting that the inhibition of migration by PLE is mainly achieved by regulating YAP activity. Taken together, the results of this study indicate that PLE suppressed cell growth and increased the apoptosis of breast cancer (BC) cells via inactivation of YAP activity in a LATS1/2-dependent manner.

\section{Introduction}

Perilla frutescens is a member of the Liliaceae/Labiatae (mint) family of flowering plants. Its leaves are consumed as a popular functional food in East Asian countries [1]. It has also been widely used as a traditional medicinal herb for expelling pathogens from the superficies and relieving exterior syndrome. The plant is used in traditional Chinese medicine (TCM) for pain due to blood stagnation caused by cold congealing. It has been used for various disorders such as depression-related diseases, asthma, anxiety, and tumors $[1,2]$.
Studies on $P$. frutescens have demonstrated its antioxidant [3], anti-inflammatory $[4,5]$, and anti-allergic properties $[5,6]$. Several studies have reported that $P$. frutescens inhibits tumor growth and invasion in metastatic cancer cell lines and animal models [7-9]. Luteolin and triterpene acids, constituents of $P$. frutescens, have anti-inflammatory and antitumor effects $[10,11]$. Although $P$. frutescens has been suggested to be a useful medicinal herb with antitumor activity, its exact molecular mechanisms are not yet fully understood.

The Hippo signaling pathway was first discovered in Drosophila, where it functions as a regulator of tissue and organ 
size [12]. Components of the Hippo pathway are highly conserved from Drosophila to humans, and a few mutations have been associated with human cancers [13]. The mammalian Hippo kinase cascade includes the mammalian STE20like1/2 (MST1/2) and large tumor suppressor kinase 1/2 (LATS1/2) as well as the downstream effector Yes-associated protein (YAP) and WW domain-containing transcription factor (TAZ) [14]. YAP and TAZ are transcription co-activators without any DNA-binding domains; they enhance transcription by binding to specific transcription factors and in turn induce the expression of genes regulating cell proliferation, apoptosis, and differentiation [15]. The Hippo pathway inhibits both YAP and TAZ in a phosphorylation-dependent manner [16]. Abnormal YAP/TAZ overexpression and dysregulation of core Hippo kinases have been frequently observed in various human cancers $[13,17]$.

Triple-negative breast cancers (TNBCs) are an aggressive type of breast cancer (BC) that do not express receptors against estrogen or progesterone and HER2 [18, 19]. These tumors have a poorer prognosis than other types of $\mathrm{BC}$ due to the resistance to hormonal therapies or HER2 targeted therapies such as trastuzumab. Thus, intense studies are being carried out seeking the underlying mechanisms and new treatment approaches for TNBCs [20]. Recent studies reported that the Hippo pathway is highly activated in TNBCs and could be a driving force for the progression of TNBCs [21-24].

Numerous proteins have been implicated to act upstream of and regulate the Hippo pathway [14, 25]. However, the medicinal herbs that regulate the Hippo pathway are mostly unknown. Therefore, the aim of this study was to investigate the antitumor effects of $P$. frutescens on TNBC cells and its role in the Hippo-YAP signaling pathway.

\section{Materials and Methods}

2.1. Reagents and Antibodies. Anti-YAP (\#14074), YAP/TAZ (\#8414), phospho-YAP (Ser127, \#4911), phospho-YAP (Ser397, \#13619), LATS1 (\#3477), LATS2 (\#5888), phosphoLATS1/2 (Ser909/Ser872,\#9157), phosphor-MST1/2 (Thr183/Thr180, \#3681), MST2 (\#3952), PARP (\#9542), Caspase-3 (\#14220), and pan-TEAD (\#13295) were purchased from Cell Signaling Technology (Beverly, MA, USA). Anti-MST1 (No. 611052) was obtained from BD Transduction Laboratories (Lexington, KY, USA). Anti-Vinculin (V9131) was obtained from Sigma-Aldrich (St. Louis, MO, USA). Normal rabbit IgG for control immunoprecipitation (sc-2027) and anti-HA (sc-7392) were purchased from Santa Cruz Biotechnology, Inc. (Santa Cruz, CA, USA). Horseradish peroxidase-conjugated secondary antibodies (NA931V and NA934V) were purchased from GE Healthcare (Chicago, IL, USA). Phos-tag-conjugated polyacrylamide was purchased from Wako Pure Chemical Industries, Ltd. (Osaka, Japan). All chemicals and solvents used were purchased from Merck (Kenilworth, NJ, USA) and SigmaAldrich, unless stated otherwise. Polyvinylidene fluoride (PVDF) membrane (Immobilon-P) was obtained from Millipore Co. (Billerica, MA, USA). SuperSignal ${ }^{\circledast}$ West Pico Chemiluminescent Substrate was obtained from Thermo Fisher Scientific, Inc. (Waltham, MA, USA).
2.2. Cell Culture and Transfection. HEK293T, LATS1/2 KO HEK293A, MST1/2 KO HEK293A, MCF7, MDA-MB-468, Hs578T, and SUM159 cells were cultured in Dulbecco's modified Eagle's medium (DMEM) containing 10\% of fetal bovine serum (FBS), 50 units $/ \mathrm{mL}$ of penicillin, and $50 \mu \mathrm{g} / \mathrm{mL}$ of streptomycin (Gibco, MA, USA). MDA-MB-231, BT549, T47D, and SKBR3 cells were cultured in RPMI1640 containing $10 \%$ FBS, 50 units $/ \mathrm{mL}$ penicillin, and $50 \mu \mathrm{g} / \mathrm{mL}$ streptomycin. MCF10A cells were cultured in DMEM/F12 containing 5\% horse serum (HS), $20 \mathrm{ng} / \mathrm{mL}$ of EGF, $0.5 \mu \mathrm{g} / \mathrm{mL}$ of hydrocortisone, $10 \mu \mathrm{g} / \mathrm{mL}$ of insulin, $100 \mathrm{ng} / \mathrm{mL}$ of cholera toxin, and 50 units $/ \mathrm{mL}$ of penicillin, and $50 \mu \mathrm{g} / \mathrm{mL}$ of streptomycin. MDA-MB-436 cells were cultured in DMEM/F12 containing $10 \%$ of FBS, 50 units $/ \mathrm{mL}$ of penicillin, and $50 \mu \mathrm{g} / \mathrm{mL}$ of streptomycin. HEK293A and MAP4K4/6/7 KO cells, kindly provided by Dr. Kun-Liang Guan's laboratory at the University of California San Diego, were maintained in DMEM with 10\% FBS [26]. The cells were grown at $37^{\circ} \mathrm{C}$ in a humidified incubator in an atmosphere of $5 \% \mathrm{CO}_{2}$. The human $\mathrm{BC}$ cell lines were purchased from ATCC (Manassas, VA) and Korean Cell Line Bank (KCLB; Seoul, South Korea). For plasmid DNA transfection, the cells were seeded on plates and incubated overnight. The cells were then transfected with a suitable concentration of each plasmid DNA using polyethyleneimine (PEI; Polysciences, Inc., Warrington, PA, USA) according to the manufacturer's instructions.

2.3. Retrovirus Infection and Stable Cell Lines. MDA-MB-231 cells were infected with retrovirus with $5 \mu \mathrm{g} /$ $\mathrm{mL}$ polybrene (Millipore Co.). Retroviral infection was performed as previously described [27]. Infected cells were selected with $2 \mathrm{mg} / \mathrm{mL}$ G418 (Gold Biotechnology, Inc., St Louis, MI, USA).

2.4. Generation of Knockout Cells. pSpCas9 (BB)-2A-Puro (PX459; plasmid \#62988) and lenti CRISPR v2 vector (plasmid \#52961) were supplied by Addgene (Watertown, MA, USA). The nucleotide guide sequences were designed using the CRISPR design tool at http://www.rgenome.net/ cas-designer/. Single-guide RNAs (sgRNAs) were cloned into the PX459 and Lenti CRISPR V2 expression vector. HEK293A cells were transfected with cloned PX459. BT549 cells were infected with lentivirus generated from Lenti CRISPR V2 vector. Transfection was performed according to the manufacturer's instructions. After $24 \mathrm{~h}$ of transfection or infection, HEK293A and BT549 cells were selected for 2 days with media containing puromycin (InvivoGen, San Diego, CA, USA). The cells were then incubated with new media for $24 \mathrm{~h}$ before single cell sorting with BD FACS Aria ${ }^{\mathrm{TM}}$ III sorter (Franklin Lakes, NJ, USA) at the Three-Dimensional Immune System Imaging Core Facility.

Guide sequences were as follows:

LATS1, 5' -GCAGCCATCTGCTCTCGTCG-3'

LATS2, 5'-GTAGGACGCAAACGAATCGC- $3^{\prime}$

MST1, 5' -TCCTGGAGGTCTGATTCCAC-3'

MST2, 5'-TGCTCCGTTCCTAAGGCAGA-3' 
2.5. Preparation of $P$. frutescens Extract. P. frutescens leaf extract (PLE) was provided by the National Institute of Horticulture Herbal Science (NIHHS; Wanju, South Korea) of this institute. PLE powder was dissolved in absolute ethanol at a concentration of $10 \mathrm{mg} / \mathrm{mL}$ and used after filtration with a $0.45 \mu \mathrm{m}$ syringe filter.

\subsection{High-Performance Liquid Chromatography (HPLC)} Assay. The concentration of polyphenolic substances in PLE was determined by HPLC. The analyses were performed using Agilent 1260 Infinity II with a ZORBAX Eclipse Plus C18 (4.6 $6250 \mathrm{~mm}, 5 \mu \mathrm{m}, 95 \AA$ A ; Agilent Technologies, Inc., Santa Clara, CA, USA). The mobile phase contained $0.1 \%$ phosphoric acid (solvent $\mathrm{A}$ ) and acetonitrile (solvent $\mathrm{B}$ ). The flow rate was $0.8 \mathrm{~mL} / \mathrm{min}$, and the injection volume was fixed at $10 \mu \mathrm{l}$. The column temperature was maintained at $35^{\circ} \mathrm{C}$ during the separation, and the optical density of the effluent was monitored at $330 \mathrm{~nm}$. Commercial $\beta$-carotene, caffeic acid, luteolin, quercetin, rosmarinic acid, and linolenic acid were purchased from Sigma-Aldrich and used as standards.

2.7. Western Blot and Immunoprecipitation Assay. Western blotting was performed using a standard protocol [27]. For immunoprecipitation, cells were lysed in a mild lysis buffer $(10 \mathrm{mM}$ Tris at $\mathrm{pH} 7.5,100 \mathrm{mM} \mathrm{NaCl}, 1 \mathrm{mM}$ EDTA, 1\% NP-40, $50 \mathrm{mM} \mathrm{NaF}, 1 \mathrm{mM} \mathrm{Na}_{3} \mathrm{VO}_{4}$, and protease inhibitor cocktail [Sigma-Aldrich]) and centrifuged at $4^{\circ} \mathrm{C}$ at $12,000 \times \mathrm{g}$. The supernatants were immunoprecipitated with appropriate antibodies for $2 \mathrm{~h}$ at $4^{\circ} \mathrm{C}$, followed by the addition of Protein A/G magnetic beads (Thermo Fisher Scientific, Inc.) for an additional $1 \mathrm{~h}$ at $4^{\circ} \mathrm{C}$. The immunoprecipitates were washed three times with the mild lysis buffer, and then the samples were eluted with SDS-PAGE sample buffer.

2.8. Luciferase Assay. HEK293A cells were seeded on 12-well plates, and after $12 \mathrm{~h}$, they were transfected with $5 \times$ UAS-Luc reporter, pRL reporter, Gal4-TEAD4, and Flag-YAP2. On the next day, the cells were treated with PLE for $10 \mathrm{~h}$ and lysed. Luciferase activity was measured using the Dual-Glo ${ }^{\circledR}$ luciferase reporter assay kit (Promega, Madison, WI, USA) according to the manufacturer's instructions. The relative firefly luciferase activity was normalized to that of Renilla luciferase.

2.9. RNA Isolation and Real-Time Quantitative Polymerase Chain Reaction (RT-qPCR). RNA isolation was performed using an RNeasy mini kit (QIAGEN, Germantown, MD, USA). Equal amounts of cDNA were synthesized from $1 \mu \mathrm{g}$ of total RNA using reverse transcriptase (Promega) with random hexamers (Takara Bio Inc., Shiga, Japan) and necessary reagents for synthesis. qPCR was performed using the SYBR Green PCR master mix (Sigma-Aldrich) with an appropriate primer pair, following the manufacturer's protocol. The normalization of several YAP-dependent genes was done using hypoxanthine-guanine phosphoribosyltransferase 1 gene (HPRT). The primers used were as follows:
HPRT, 5'-AGAATGTCTTGATTGTGGAAGA/ACCTTGACCATCTTTGGATTA-3'

CTGF, 5'-CCAATGACAACGCCTCCTG/TGGTGCAGCCAGAAAGCTC- $3^{\prime}$

CYR61, 5'-AGCCTCGCATCCTATACAACC/TTCTTTCACAAGGCGGCACTC-3'

INHIBA, 5'-TTGCCAACCTCAAATCGTGCT/CCCACACTCCTCCACGATCAT-3'

CXCL1, 5'-CCACTGAACTGCGCTGCCA/AGCTTTCCGCCCATTCTTGAG-3'

FSTL1, 5'-GCAGCAACTACAGTGAAATCC/ATGGCAGTTTCATTCTGTTCC- $3^{\prime}$

PAPPA, 5 '-TCTTGGTCACTGATGGTGTGA/AGAGGTCTCCGGCAGTGAT-3'

2.10. Wound Healing Assay. For the wound healing assay, the cells were plated in 6-well plates and allowed to reach approximately $80 \%$ confluence. Wounds were then generated by scratching the surface using a Scar ${ }^{\mathrm{TM}}$ scratcher (SPL Life Sciences Co., Ltd., South Korea), followed by washing with phosphate-buffered saline (PBS) to remove the cell debris. The cell medium was replenished with or without PLE and allowed to migrate to the wound area for $36 \mathrm{~h}$. The wound regions were photographed every $12 \mathrm{~h}$, and the images were captured under a phase-contrast microscope (OLYMPUS 1X71 DP controller, Tokyo Japan). The average wound widths at 0 and $36 \mathrm{~h}$ time points were quantified using the Image program (National Institutes of Health, Bethesda, MD, USA).

2.11. Cytotoxicity Assay. Cell viability was assessed using the EZ-Cytox cell viability assay kit (Daeil Lab Service, Seoul, South Korea). Briefly, HEK293A, MCF10A, MDAMB-231, and BT549 cells were added to each well of a 96well plate. After incubation for $24 \mathrm{~h}$, the cells were treated with PLE for $24 \mathrm{~h}$. Then, $10 \mu \mathrm{L}$ EZ-Cytox solution was added to each well of the plate and the cells were incubated at $37^{\circ} \mathrm{C}$ for $2 \mathrm{~h}$. Subsequently, the absorbance was measured at $450 \mathrm{~nm}$ using a microplate spectrophotometer (BioTek Instruments, Winooski, VT, USA). Cell viability (\%) was calculated using the formula $\left[A_{s} / A_{c}\right] \times 100$, where $A_{s}$ is the absorbance of the well-containing cells, culture medium, EZ-Cytox solution, and stimulants and $A_{c}$ is the absorbance of the well-containing cells, culture medium, and EZ-Cytox solution.

2.12. Sulforhodamine B (SRB) Assay. HEK293A, MCF10A, MDA-MB-231, and BT549 cells were seeded into each well of a 96-well plate. After incubation for $24 \mathrm{~h}$, the cells were treated with DMSO and $100 \mu \mathrm{g} / \mathrm{mL}$ of PLE for up to 5 days; the cell medium with serially diluted PLE was refreshed every 2 days. The plates were harvested every 2 days at the same time and fixed with $10 \%$ trichloroacetic acid (TCA) solution (Sigma-Aldrich) at $4^{\circ} \mathrm{C}$ overnight. The plates were washed with distilled water more than three times. After that, the plates were stained with $0.4 \%$ SRB (Sigma-Aldrich) 
in $1 \%$ acetic acid solution for $30 \mathrm{~min}$ at $25^{\circ} \mathrm{C}$. The cells were then washed with $0.1 \%$ acetic acid solution and dried at $25^{\circ} \mathrm{C}$ for $1 \mathrm{~h}$. Finally, $10 \mathrm{mM}$ Trizma base (Sigma-Aldrich) was added, and the plates were incubated on a rocker for $1 \mathrm{~h}$ to resolve the stained cells. The SRB levels were measured using a microplate spectrophotometer (BioTek Instruments) at an absorbance of $540 \mathrm{~nm}$.

2.13. Clonogenic Growth Assay. MDA-MB-231 cells and TEAD1 $\triangle \mathrm{C}-\mathrm{YAP}(\mathrm{AD})$-expressing cells were seeded in a 6well plate. After $24 \mathrm{~h}$, the cells were treated with 10 and $25 \mu \mathrm{g} / \mathrm{mL}$ of PLE for 10 days. Then, the cells were fixed with $4 \%$ formaldehyde for $10 \mathrm{~min}$ and stained with $0.25 \%$ crystal violet for $12 \mathrm{~h}$. After washing three times with distilled water, the cells were destained with $95 \%$ ethanol for $1 \mathrm{~h}$. The absorbance of the $100 \mu \mathrm{L}$ destaining solution was measured at $595 \mathrm{~nm}$ using a microplate spectrophotometer (BioTek Instruments).

2.14. Statistical Analysis. Values are expressed as the mean \pm standard error of the mean (SEM) of three samples obtained from three independent experiments. Student's $t$ test (unpaired, one-tailed) was performed to calculate a $p$-value using Prism 5.0 software (GraphPad Software, La Jolla, CA, USA) and considered at ${ }^{*} p<0.05,{ }^{* *} p<0.01$, and ${ }^{* * *} p<0.001$

\section{Results}

3.1. Analysis of PLE using HPLC. In this study, we screened medicinal herb extracts to identify novel inhibitors of YAP/ TAZ. Through a review of the literature, 13 medicinal herbs were selected to screen for possible inhibitory activity against YAP/TAZ through the Hippo tumor suppressor pathway. To determine the effects of the medicinal herb on YAP activation, we evaluated the YAP phosphorylation status using mobility shift on a phos-tag gel. A pilot screening of the medicinal plant extracts identified that PLE potently inhibited YAP by promoting its phosphorylation (Figure S1).

Phytochemical constituents such as catechin, ferulic acid, apigenin, luteolin, rosmarinic acid, and caffeic acid have been identified from $P$. frutescens [28]. Therefore, in this study, commercially available $\beta$-carotene, caffeic acid, luteolin, quercetin, rosmarinic acid, and linolenic acid were used as standard compounds for HPLC-UV analysis. The quantitative profiles of the PLE composition are shown in Figure 1(a). Rosmarinic acid was exclusively detected in the range of $38-40 \mathrm{~min}$ and at $4.6 \mathrm{mg}$ per gram of PLE (Figure 1(b) and Table 1).

3.2. PLE Increases YAP Phosphorylation and Reduces YAPTEAD-Mediated Transcriptional Activity. To investigate the molecular mechanisms associated with the Hippo-YAP pathway, we treated HEK293A cells with the PLE. The IC50 value of HEK293A was $584.3 \mu \mathrm{g} / \mathrm{mL}$ (Figure $2(\mathrm{a})$ ), and the percentages of cell proliferation were $18.6 \%$ lower when treated with $100 \mu \mathrm{g} / \mathrm{mL}$ of PLE, compared to those with control (Figure 2(b)). Expression of pYAP, YAP, and TAZ in PLE-treated HEK293A cells was determined by immunoblotting. As shown in Figure 2(c), HEK293A cells showed increased phosphorylation of YAP at Ser127 and Ser397 following PLE treatment. Activated LATS1/2-driven phosphorylation on both Ser127 and Ser397 is an important step for nuclear/cytosol translocation and protein degradation $[16,29]$. PLE significantly reduced YAP and TAZ levels in HEK293A cells in a concentration-dependent manner. These results are consistent with those of previous studies showing that phosphorylated YAP and TAZ are sequestered and degraded in the cytoplasm, thus revealing that PLE could suppress the function of YAP by upregulating the level of phosphorylation [30].

Many transcription factors, including TEA domain family members (TEADs), RUNX2, TBX5, Oct4, and ErbB4, have been implicated as YAP partners [31, 32]. In mammalian cells, the TEADs (TEAD1-4) are major partners mediating YAP/TAZ biological functions [33]. When Hippo signaling is not activated, YAP and TAZ are not phosphorylated. These unphosphorylated YAP and TAZ enter the nucleus and bind to the transcription factor TEAD to induce gene transcription [34]. To determine the effects of PLE on YAP/TAZ and TEAD binding, we conducted a coimmunoprecipitation assay in HEK293A cells. Consistent with YAP phosphorylation, PLE blocked the interaction between YAP and TEAD (Figure 2(d)). To ascertain the effects of PLE on the YAP-TEAD transcriptional complex, we evaluated its transcriptional activity using the luciferase reporter system (Figure 2(e)). PLE also effectively blocked the ability of YAP to activate TEAD-mediated transcription but did not abolish its ability to activate RUNX2 (Figure S2).

To further confirm the negative regulation of YAP by PLE, we measured YAP-TEAD-driven target gene expression. We found that PLE significantly diminished the expression of YAP-TEAD target genes, including CTGF, CYR61, INHBA, CXCL1, FSTL1, and PAPPA, in a dosedependent manner (Figure 2(f)). Collectively, our data suggest that PLE inhibited YAP activity via disruption of the YAP-TEAD transcriptional complex.

3.3. PLE Inhibits YAP/TEAD Transcriptional Activity via LATS1/2-Dependent Mechanisms but Not MST1/2-Dependent Mechanisms. Among the Hippo signaling core kinases that regulate YAP/TAZ activity, LATS1/2 is one of the most significant kinases for YAP. Phosphorylation of Ser909 and Thr1079 is essential for LATS1 kinase activity [35]. Following PLE treatment, the increase in YAP phosphorylation could be due to LATS1 kinase activity. To test this hypothesis, we monitored Ser909 phosphorylation in endogenous LATS1 in response to PLE. Phosphorylation of Ser909 in LATS1 was increased by PLE treatment (Figure 3(a)). Next, we attempted to determine whether PLE downregulates the transcriptional activity of YAP/TAZ via LATS $1 / 2$ using the LATS1/2 ${ }^{-1-}$ (LATS1/2 KO) HEK293A cells generated by the CRISPR-Cas9 system Figure 3(b). The transcriptional activation of YAP target genes such as CTGF 


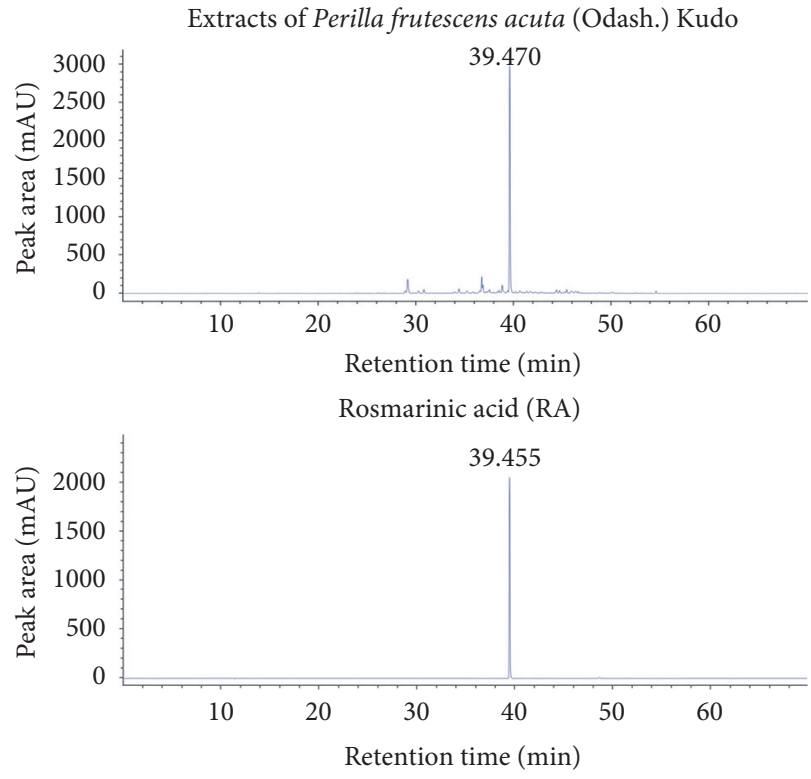

(a)<smiles>O=C(/C=C/c1ccc(O)c(O)c1)OC(Cc1ccc(O)c(O)c1)C(=O)O</smiles>

(b)

Figure 1: Flavonoid profiles of the extracts of Perilla frutescens var. acuta (Odash.) Kudo leaves (PLE). (a) Comparative high-performance liquid chromatography ultraviolet (HPLC-UV) chromatograms of PLE ethanol extracts (top) and rosmarinic acid (RA, bottom) in the standard solution were obtained using an Agilent 1260 series liquid chromatography system. Chromatographic separation was carried out on a ZORBAX Eclipse Plus C18 column $(4.6 \times 150 \mathrm{~mm}, 5 \mu \mathrm{m})$, and the column temperature was maintained at $35^{\circ} \mathrm{C}$. The mobile phase consisted of water containing $0.1 \%$ phosphoric acid (A) and acetonitrile (B). The composition of the mobile phase was $0-4$ min, $0 \%$ (B); $10 \mathrm{~min}, 4 \%$ (B); $20 \mathrm{~min}, 10 \%$ (B); $30 \mathrm{~min}, 20 \%$ (B); $40 \mathrm{~min}, 40 \%$ (B); and $70 \mathrm{~min}, 100 \%$ (B). RA was detected at concentrations ranging from 38 to $40 \mathrm{~min}$. PLE contained $4.6 \mathrm{mg} / \mathrm{g}$ of RA. (b) The chemical structure of RA isolated from PLE.

TABle 1: Retention time, calibration equation, and correlation coefficient for compound in extracts of Perilla frutescens var. acuta (Odash.) Kudo leaves.

\begin{tabular}{lclr}
\hline Compound & Retention time $(\mathrm{min})$ & Calibration equation & Correlation coefficient $\left(r^{2}\right)$ \\
\hline Rosmarinic acid & 39.455 & $Y=57.769 x+215.250$ & 0.999 \\
\hline
\end{tabular}

a: peak area, $x$ : concentration of standards $(\mathrm{mg} / \mathrm{mL})$.

and CYR61 was suppressed by PLE treatment. However, no noticeable changes in the expression levels of YAP target genes were observed between PLE-treated and untreated groups in LATS1/2 KO cells (Figure 3(c)). This suggests that PLE regulates target gene expression via LATS1/2-dependent mechanisms.

MST1/2 is autophosphorylated on multiple sites in response to various stress stimuli, which results in the activation of MST1/ 2 [36]. Among these multiple autophosphorylation sites, phosphorylation at Thr183 and Thr180 is essential for Mst1 and Mst2 activation, respectively [37]. Following PLE treatment, the increase in LATS1/2 phosphorylation could be due to MST1/2 activation. To test this hypothesis, we monitored Thr183 phosphorylation. The phosphorylation of Thr183 in MST1 was slightly increased by PLE treatment (Figure 3(d)). Next, to confirm the role of MST1/2 in PLE-induced YAP regulation, we compared YAP target gene expression between wild-type and MST1/2 $2^{-1-}$ (MST1/2 KO) HEK293A cells (Figure 3(e)). As expected, PLE treatment decreased the expression of CTGF and CYR61 (Figure 3(f)). However, PLE still reduced the expression of CTGF and CYR61 in MST1/2 KO cells, suggesting that PLE reduces YAP target gene levels independent of MST1/2.
Although MST1/2 is well characterized as an upstream activating kinase of LATS1/2 [35], accumulating evidence indicates that MST1/2 and MAP4Ks directly phosphorylate and activate LATS1/2 in a partially redundant manner $[26,38,39]$. Next, to confirm the role of MAP4Ks in PLE-induced YAP regulation, we compared YAP target gene expression between wild-type and MAP4K4/6/7 ${ }^{-1}$ (MAP4K4/6/7 KO) HEK293A cells. PLE treatment decreased the expression levels of CTGF and CYR61, but MAP4K4/6/7 deletion did not alter the expression levels of these genes in response to PLE treatment, suggesting that PLE could reduce YAP target gene levels dependent on MAP4K4/6/7 (Figure S3). In this study, we discovered a novel LATS1/2-dependent mechanism for the inhibitory effect of PLE on YAP/TAZ phosphorylation and inactivation, but MAP4Ks had a redundant function in activating LATS1/2 kinases.

3.4. Induction of Cytotoxicity and Apoptosis by PLE in TNBC Cells $M D A-M B-231$ and BT549. The Hippo-YAP signaling pathway has attracted much attention in cancer research since the Hippo-YAP signaling pathway, a well-known regulator of organ size, has been known to play an essential 


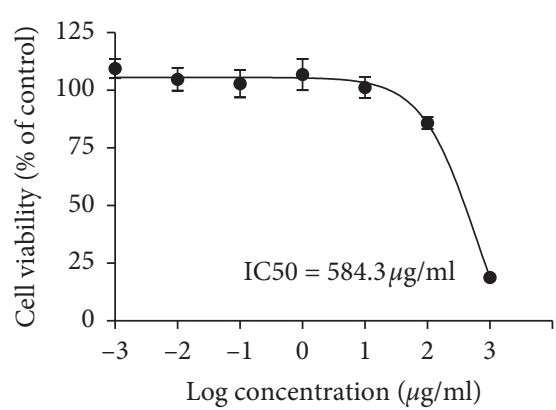

(a)

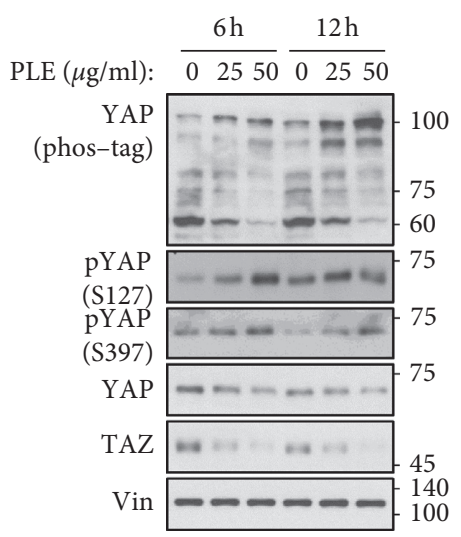

(c)

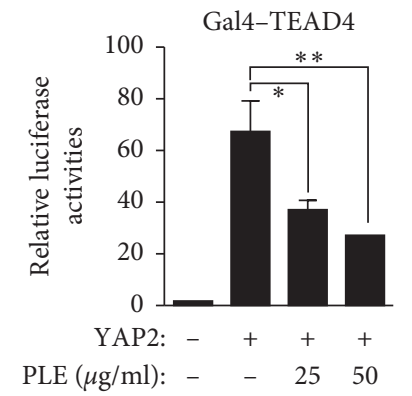

(e)

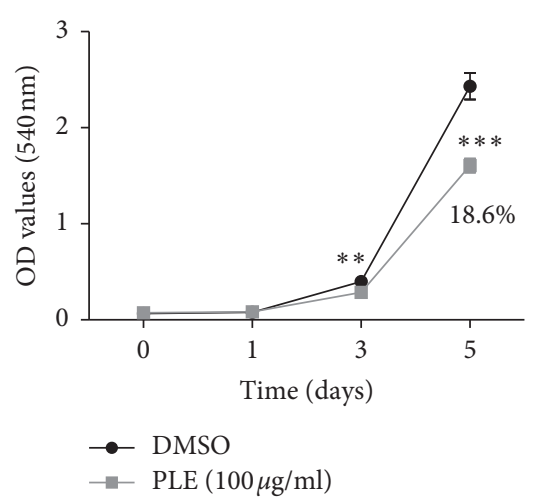

(b)

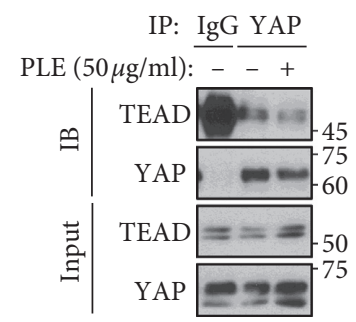

(d)

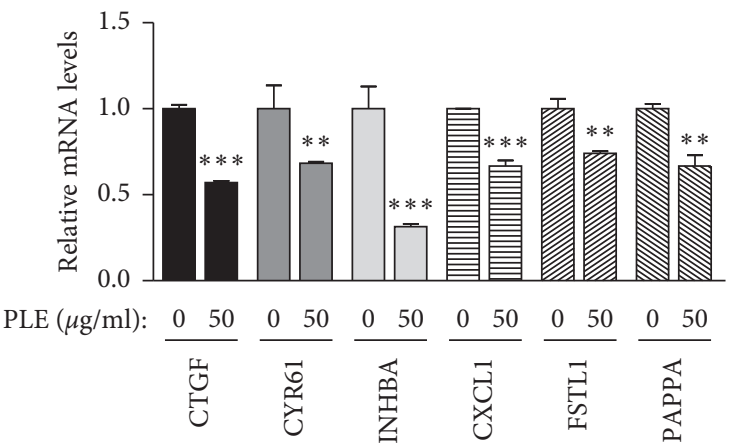

(f)

FIgURE 2: PLE inhibits YAP activity and disrupts YAP-TEAD complex to inhibit its transcriptional activity. HEK293A cells were treated using the indicated concentrations of PLE for $24 \mathrm{~h}$. (a) Cell viability was measured using an EZ-Cytox cell viability assay kit. IC50 values were calculated from concentration response curves using Prism 5.0 software. The IC50 value of HEK293A cells was $584.3 \mu \mathrm{g} / \mathrm{mL}$. Error bars represent \pm SEM from $n=3$ per group. (b) HEK293A cells were treated with DMSO and $100 \mu \mathrm{g} / \mathrm{mL}$ of PLE for 5 days, and cell proliferation was measured by SRB assay at an absorbance of $540 \mathrm{~nm}$. The proliferation of HEK293A cells with PLE was decreased by $18.6 \%$ compared with control cells. Error bars represent mean $\pm \operatorname{SEM}(n=3)$. ${ }^{* * *} p<0.001,{ }^{* *} p<0.01$; Student's $t$-test (unpaired, one-tailed) was used for statistical analysis. (c) HEK293A cells were treated with various concentrations (50 and $100 \mu \mathrm{g} / \mathrm{mL}$ ) of PLE for 2 and $6 \mathrm{~h}$, YAP phosphorylation states were determined by phos-tag gel shift assay, and cell lysates were subjected to immunoblotting with anti-YAP, pYAP, TAZ, and Vin. (d) HEK293A cells were treated with PLE $(50 \mu \mathrm{g} / \mathrm{mL})$ for $6 \mathrm{~h}$. Endogenous YAP/TAZ was immunoprecipitated, and the coprecipitated pan-TEAD was detected by western blot. (e) HEK293A cells were co-transfected with $5 \times$ UAS-luciferase reporter, Gal4TEAD4, Renilla, with or without HA-YAP2. HEK293A cells were treated with PLE in a concentration-dependent manner. Cells were harvested $10 \mathrm{~h}$ after treatment. Representative results of a single experiment with $n=3$ biological replicates; three independent experiments were carried out. (f) HEK293A cells were treated with $50 \mu \mathrm{g} / \mathrm{mL}$ of PLE for $12 \mathrm{~h}$ before harvesting. mRNA levels of CTGF, CYR61, INHBA, CXCL1, FSTL1, and PAPPA were determined using RT-qPCR. Bars represent mean \pm SEM $(N=3) .{ }^{*} p<0.05,{ }^{* *} p<0.01$, and ${ }^{* * *} p<0.001$; Student's $t$-test (unpaired, one-tailed) was used for statistical analysis. 


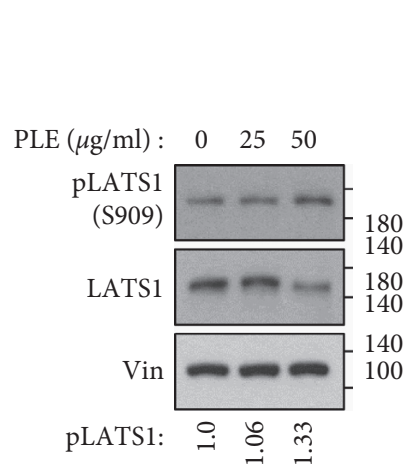

(a)

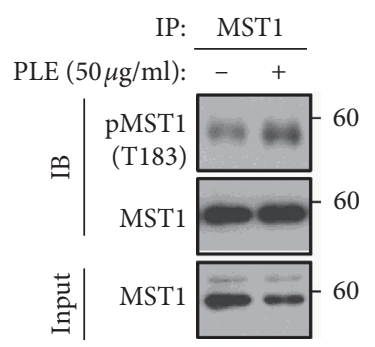

(d)

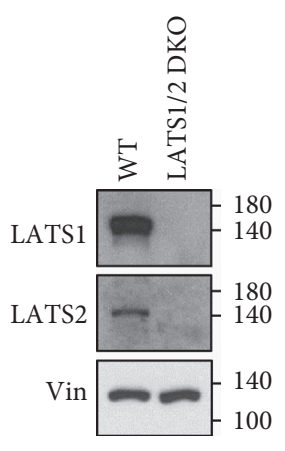

(b)

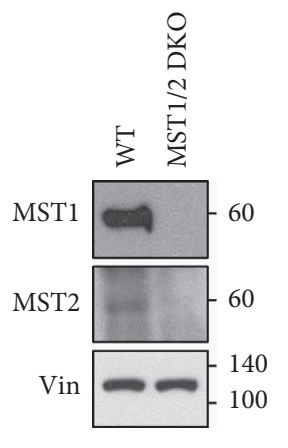

(e)
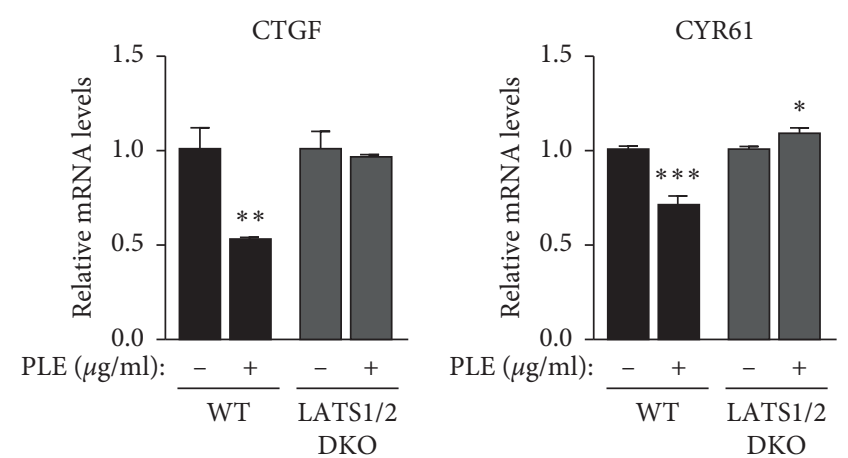

(c)
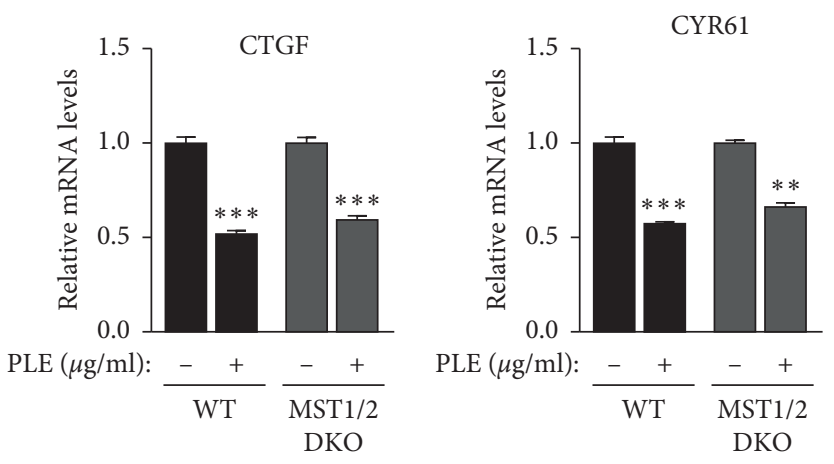

(f)

FIGURE 3: PLE suppressed YAP target genes in a LATS1/2-dependent manner. (a) HEK293A cells were treated with increasing concentrations of PLE for $2 \mathrm{~h}$. Cell lysates were immunoblotted using anti-LATS1 and anti-pLATS1 (S909) antibodies. Quantifications of western blots for pLATS1 (S909) were performed by ImageJ program. (b, c) Wild-type and LATS1/2 knockout (KO) HEK293A cells were treated with PLE. After $12 \mathrm{~h}$, mRNA levels of CTGF and CYR61 were measured using RT-qPCR. Error bars represent \pm SEM from $n=3$ per group. (d) HEK293A cells were treated with increasing concentrations of PLE for $2 \mathrm{~h}$. Cell lysates were immunoblotted using anti-MSTS1 and anti-pMST1 (T183) antibodies. (e,f) Wild-type and MST1/2 KO HEK293A cells were treated with PLE for $12 \mathrm{~h}$ and mRNA levels of CTGF and CYR61 were measured using RT-qPCR (error bars represent \pm SEM from $n=3$ per group). Cell lines were immunoblotted to analyze indicated antibodies. ${ }^{*} p<0.05,{ }^{* *} p<0.01$, and ${ }^{* * *} p<0.001$; Student's $t$-test (unpaired, one-tailed) was used for statistical analysis In Figure $3(\mathrm{a})$ ), the numbers of size maker depart from indicated lines.

role in cancer development $[40,41]$. The regulation of Hippo-YAP represents a promising strategy for the treatment of various cancers [21, 42]. Therefore, we further explored the effects of PLE on Hippo-YAP signaling in TNBC cells. Western blotting was performed to determine the levels of YAP and TAZ protein in $10 \mathrm{BC}$ cell lines. YAP and TAZ were overexpressed in the MDA-MB-231, BT549, and MDA-MB-468 cell lines, whereas their expression levels were relatively low in the MCF7 and MDA-MB-436 cell lines. MDA-MB-231 and BT549 have been reported to have high invasion potential; therefore, these two cell lines were selected for further study (Figure S4).

To investigate whether PLE has a better cytotoxic effect on TNBC compared to normal human mammary epithelial cell line, the cytotoxic activity of PLE was examined using MTT assay in MDA-MB-231, BT549, and MCF10A cells with various concentrations of PLE for $24 \mathrm{~h}$. The results showed that PLE significantly inhibited the growth of MDA-MB-231 and BT549 cells to a greater extent compared with that of MCF10A cells in a concentration-dependent manner, with IC50 values of $268.9 \mu \mathrm{g} /$ $\mathrm{mL}$ (Figure 4(a)), $307.1 \mu \mathrm{g} / \mathrm{mL}$ (Figure 4(d)), and $680.5 \mu \mathrm{g} / \mathrm{mL}$ (Figure S5(a)), respectively. The IC50 values showed that
MCF10A cells were more resistant to the cytotoxic effect of PLE than both TNBC cells. These results showed that PLE has a stronger cytotoxic effect on TNBC cell lines than on nonmalignant MCF10A cells. We then examined the effect of PLE on cell apoptosis. Because the cleavage of several key proteins, such as Caspase- 3 and PARP, is considered to be a hallmark of apoptosis, we measured the levels of cleaved Caspase- 3 and PARP in MDA-MB-231 and BT549 cells at PLE concentrations of 50 and $100 \mu \mathrm{g} / \mathrm{mL}$. Western blot analysis revealed significant upregulation of cleaved Caspase- 3 and PARP in both BC cell lines in a dose-dependent manner (Figures 4(b) and 4(e)).

Since PLE decreased TNBC cell viability and increased cell apoptosis effectively, we next investigated the suppressive effect of PLE on TNBC cell proliferation. MCF10A, MDA-MB-231, and BT549 cells were treated with $100 \mu \mathrm{g} / \mathrm{mL}$ PLE for 5 days, and their proliferation ability was assessed using the SRB assay. As shown in Figures 4(c) and 4(f), PLE markedly inhibited the proliferation of MDA-MB-231 and BT549 cells to a greater extent compared with that of MCF10A cells (Figure S5(b)). Collectively, these results indicate that PLE significantly inhibited the growth of MDAMB-231 and BT549 cells. 


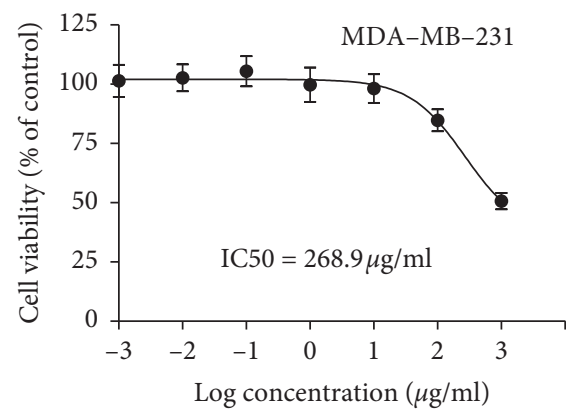

(a)

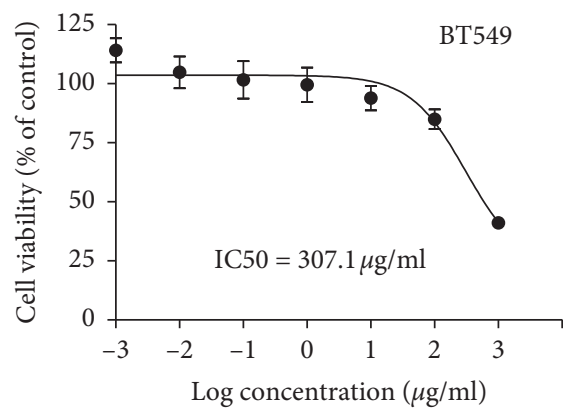

(d)

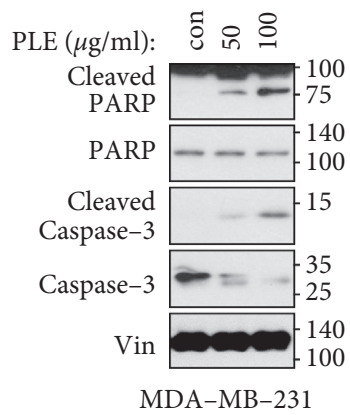

(b)

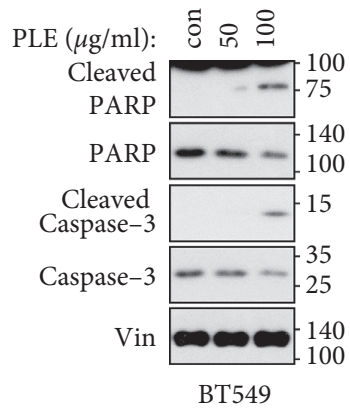

(e)

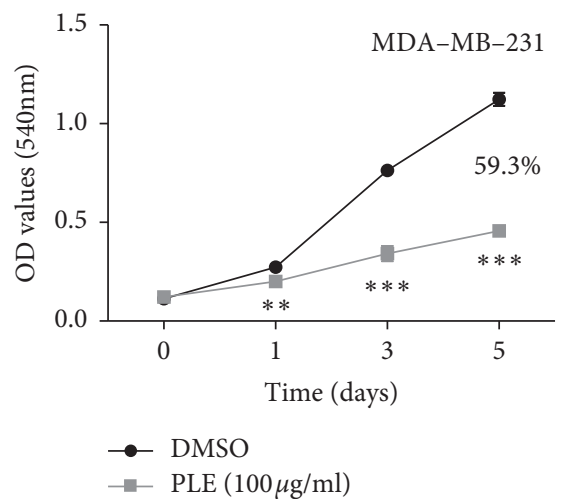

(c)

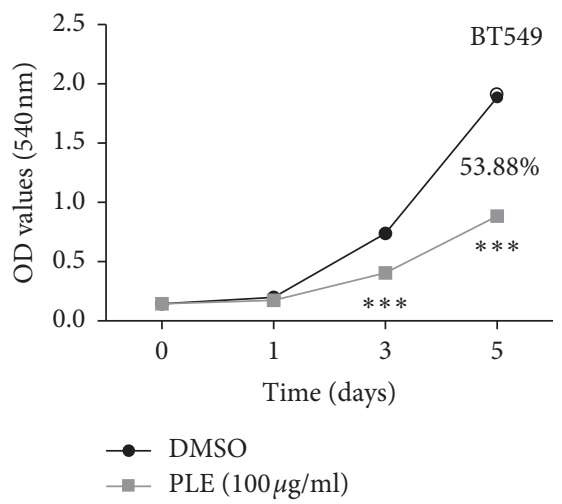

(f)

Figure 4: Cytotoxic, apoptosis-inducing, and antiproliferative effects of PLE on MDA-MB-231 and BT549. (a, d) MDA-MB-231 and BT549 cells were treated with various concentrations $(0.001,0.01,0.1,1,10,100$, and $1000 \mu \mathrm{g} / \mathrm{mL})$ of PLE for $24 \mathrm{~h}$. Cell viability was measured by using the EZ-Cytox cell viability assay kit. IC50 values were determined from concentration response curves using Prism 5.0 Software. The IC50 value of MDA-MB-231 and BT549 cells was $268.9 \mu \mathrm{g} / \mathrm{ml}$ and $307.1 \mu \mathrm{g} / \mathrm{ml}$, respectively. Error bars represent \pm SEM from $n=3$ per group. (b, e) To measure the levels of apoptosis-related protein, western blot was performed on MDA-MB-231 and BT549 cells treated with the PLE at 50 and $100 \mu \mathrm{g} / \mathrm{mL}$ for $12 \mathrm{~h}$. The lysates were immunoblotted with anti-PARP, cleaved PARP, Caspase-3, and cleaved Caspase-3. (c, f) MDA-MB-231 and BT549 cells were treated with DMSO and $100 \mu \mathrm{g} / \mathrm{mL}$ PLE, and the medium with diluted PLE was refreshed every two days. After treatment for another 5 days, cell proliferation was determined by using SRB assay at an absorbance of $540 \mathrm{~nm}$. The proliferation of MDA-MB-231 and BT549 cells with PLE was decreased by $59.3 \%$ and $53.88 \%$, respectively, compared with control cells. Bars represent mean \pm SEM $(n=3) .{ }^{* * *} p<0.001,{ }^{* *} p<0.01$; Student's $t$-test (unpaired, one-tailed) was used for statistical analysis.

3.5. PLE Blocks Tumor Progression of Human BC Cells by Repressing YAP Activity. We further explored the effects of PLE on Hippo-YAP signaling in TNBC cells. We examined the levels of phosphorylated YAP in treated BT549 and MDA-MB-231 cells. As shown in Figures 5(a) and 5(b), PLE induced phosphorylation and degradation of YAP as well as TAZ in MDA-MB-231 and BT549 cells in a dose-dependent manner. At least one TEAD gene is expressed in most adult tissues [43-45], and all four TEADs are abundantly expressed in TNBC cells as indicated by the expression profiles of the TEAD extracted from the Cancer Cell Line Encyclopedia (CCLE) (Figure S6). To determine the effects of PLE on YAP/TAZ and TEAD binding, we conducted a co-immunoprecipitation assay in MDA-MB-231 cells. Consistent with YAP phosphorylation, PLE blocked the interaction between YAP and TEAD (Figure 5(c)), strengthening the notion of the link between PLE and YAP inhibition. YAP enhances several processes responsible for tumor growth and metastasis, such as cellular proliferation, migration, and invasion [46, 47]. Therefore, we performed a wound-healing assay to determine whether PLE inhibits the migration of MDA-MB-231 cells. As expected, PLE suppressed the migration of MDA-MB-231 cells (Figure 5(d)). To ascertain the effects of PLE on the YAP-TEAD transcriptional complex and thereby confirm whether the inhibition of migration by PLE was due to the inhibition of YAP-TEAD, we constructed MDA-MB-231 cells expressing an active YAPTEAD fusion protein (TEAD1 $\triangle \mathrm{C}$-YAP $(\mathrm{AD})$ ) (Figure $\mathrm{S} 7)$. The migration inhibitory effect of PLE disappeared in TEAD $\Delta \mathrm{C}$ YAP (AD)-expressing MDA-MB-231 cells. This result suggests that inhibition of migration by PLE is achieved by inhibiting YAP-TEAD activity (Figure 5(d)). Further, to evaluate the biological effects of PLE on the growth of MDA-MB-231 cells, we performed a clonogenic growth assay in both the vector and TEAD1 $\triangle$ C-YAP(AD) expressing MDA-MB-231 cells. The ability of PLE to inhibit cell growth also disappeared in MDAMB-231 cells expressing TEAD1 $\triangle \mathrm{C}-\mathrm{YAP}(\mathrm{AD})$ (Figure 5(e)). Collectively, these results demonstrate that PLE suppressed tumor progression by modulating YAP activity.

These findings were further confirmed by examining the cytotoxic activity and the level of cleaved PARP1 in 


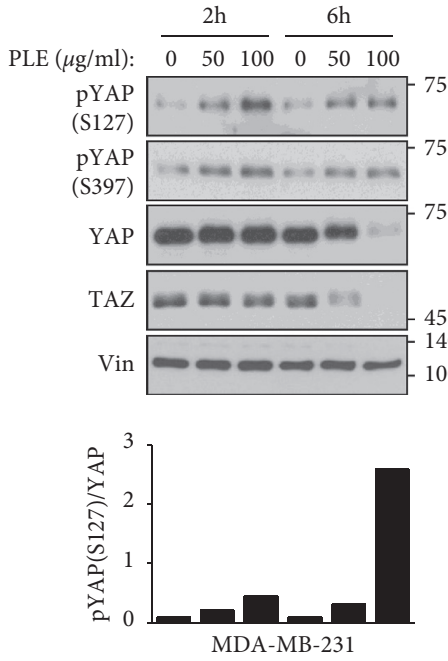

(a)

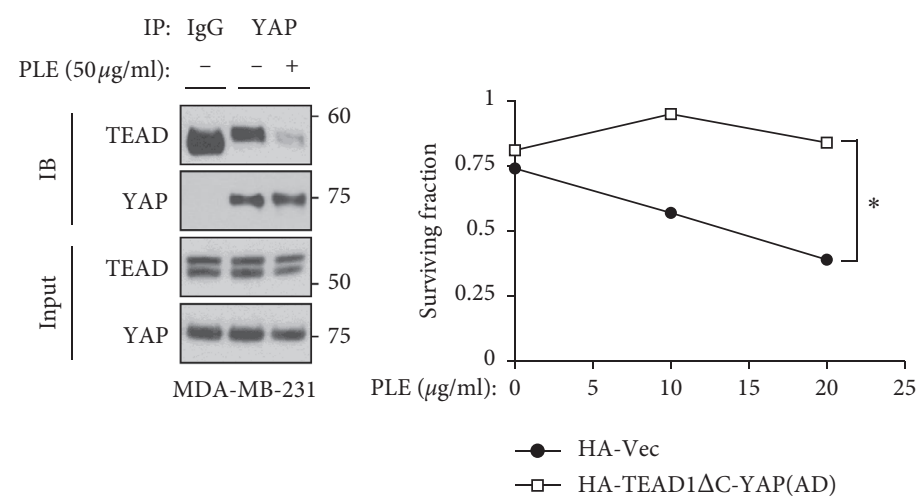

(c)

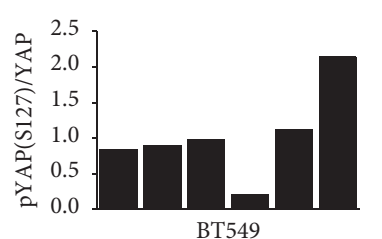

(b)
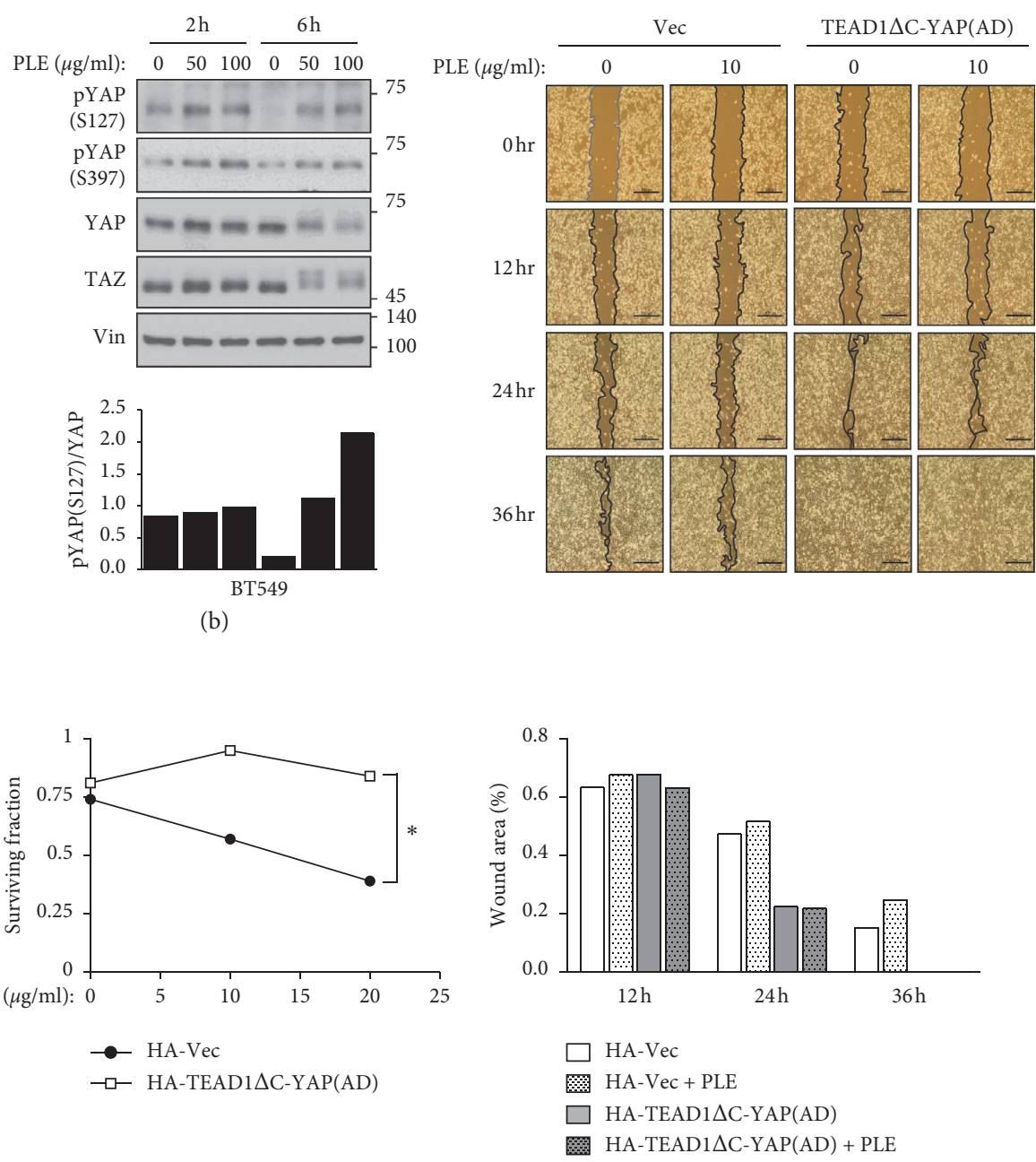

(d)

Figure 5: Constitutively active YAP-TEAD rescued PLE-induced inhibition of cancer cell migration and proliferation. (a, b) To determine the effect of PLE on the YAP phosphorylation, MDA-MB-231 and BT549 cells were treated with PLE (25 and $50 \mu \mathrm{g} / \mathrm{mL})$ for 6 and $12 \mathrm{~h}$. Cell lysates were subjected to immunoblotting with the indicated antibodies. The bar graphs show the pYAP (S127) amounts normalized to total YAP amounts for individual samples. (c) HEK293A cells were treated with PLE $(50 \mu \mathrm{g} / \mathrm{mL})$ for $6 \mathrm{~h}$. Endogenous YAP/TAZ was immunoprecipitated, and the co-precipitated pan-TEAD was detected by western blot. (d) Mobility of MDA-MB-231 cells expressing the HAvector or TEAD1 $\triangle \mathrm{C}$-YAP $(\mathrm{AD})$ was evaluated using the wound healing assay. The cells were wounded and treated with PLE $(10 \mu \mathrm{g} / \mathrm{mL})$ for $36 \mathrm{~h}$ in a serum-containing medium. At different time points $(0,12,24$, and $36 \mathrm{~h})$, phase-contrast photographs of the wounds were taken and analyzed using ImageJ program. (e) MDA-MB-231 cells expressed with HA-vector or TEAD1 $\triangle \mathrm{C}$-YAP (AD) were treated with PLE at concentrations of 10 and $25 \mu \mathrm{g} / \mathrm{mL}$. The cells were applied to clonogenic growth assay after 10 days of the treatment. In the clonogenic growth assay, DMSO-treated cells served as a control. Each value is expressed as mean \pm SEM from $n=3$ per group. ${ }^{*} p<0.05,{ }^{* *} p<0.01$; Student's $t$-test (unpaired, one-tailed) was used for statistical analysis. In Figure 5(b), the numbers of size maker depart from indicated lines.

BT549 WT and LATS1/2 KO cells generated by the CRISPR-Cas9 system (Figure 3(b)). The MTT assay showed that BT549 LATS1/2 KO cells were more resistant to the cytotoxicity of PLE than BT549 WT cells after treatment with PLE at increasing concentrations for $24 \mathrm{~h}$. The IC50 value of BT549 WT was $307.1 \mu \mathrm{g} / \mathrm{mL}$ (Figure 4(d)), and the BT549 LATS1/2 KO showed an IC50 value of $713 \mu \mathrm{g} / \mathrm{mL}$ (Figure S8(a)). Western blot results showed that PLE increased the levels of cleaved PARP1 proteins in BT549 cells to a greater extent compared with those in BT549 LATS1/2 KO cells (Figure S8(b)). These findings imply that PLE requires LATS1/2 to repress YAP/TAZ signaling.

\section{Discussion}

$\mathrm{BC}$ is the most frequently occurring cancer among women, accounting for $25 \%$ of all cancer cases worldwide. Despite the substantial efforts made in the past decades to improve survival and quality of life, BC remains a deadly threat for patients [48]. TNBCs, characterized by the absence of estrogen, progesterone, and ErbB2 receptors, account for approximately $10-15 \%$ of all $\mathrm{BCs}$ and are an aggressive type of BC. They have a poorer prognosis than other types of BCs. TNBC has an inadequate response to tamoxifen or trastuzumab, which has been developed to treat estrogen receptor-positive (ER+) BC or HER2 positive $\mathrm{BC}[49,50]$. In addition to the limitations of targeted 
therapies, the appearance of drug resistance to monotherapy is a major obstacle to the treatment of TNBC [18-20]. Thus, there is a continuing need to explore new therapeutic targets in BC. In addition, the discovery of therapeutic agents for these targets is still essential in chemotherapy for BC.

The role of YAP in organ size control or tumor development has been confirmed in mammals using transgenic mouse models $[12,51]$. YAP and TAZ are cotranscription factors between the cytoplasm and nucleus. They are known to induce cell proliferation and antiapoptotic genes by binding to several transcription factors, particularly TEADs [41]. When Hippo, the core upstream kinase of YAP/TAZ, is activated, it phosphorylates LATS1/2, which in turn inactivates YAP/TAZ via phosphorylation. Conversely, when the Hippo kinase system is off, unphosphorylated YAP/TAZ enters the nucleus and expresses target genes associated with cell proliferation [34].

Activation of YAP/TAZ has been reported in various cancers $[13,52]$. YAP/TAZ plays a particular role in the transformation of healthy epithelial cells into metastasized cancer cells through the EMT process [53]. In particular, YAP/TAZ activity is proportionally correlated with the grade and stage of BC [54], and nuclear expression of TAZ is strongly associated with TNBC [55]. YAP enhances tumor progression and metastasis in BC cells [47]. Thus, YAP/TAZ has an oncogenic role in the migration and invasion of $\mathrm{BC}$ cells [56]. Strategies to inhibit YAP/TAZ activity or activate Hippo signaling can be beneficial in treating various cancers, including TNBC.

Natural medicinal products are derived from living organisms, such as plants, animals, or microorganisms. Recently, natural compounds have gained importance with an improved understanding of their pharmacological or biological effects and contributions for medicinal use [57]. Clinical studies have revealed that natural compounds have considerable promise against many diseases [58]. Thus, natural compounds with physiological functions have been identified as targeted interventions that can regulate the activity of key molecules of signaling pathways. Among the numerous natural plant products, $P$. frutescens has been widely used as a source of herbal materials in TCM. PLE is a functional and medicinal herb that has been widely consumed in Asian countries. Previous studies have reported that PLE has anti-oxidative, anti-inflammatory, and antitumor effects [2-7]. Several studies have revealed the presence of anthocyanins, flavonoids, and phenolic acids in $P$. frutescens [3, 8-11]. In addition, recent studies suggest that various compounds in PLE have anticancer activities. Luteolin, a flavonoid isolated from $P$. frutescens leaves, has been reported to inhibit tumor proliferation and metastasis and induce apoptosis via various cellular signaling pathways [59-61]. Rosmarinic acid was suggested as an active ingredient mediating anticarcinogenic effects in a mouse skin papilloma model and exerting anti-inflammatory effects through its antioxidant activity [62-64].

Our results showed that PLE exerts an anticancer effect on MDA-MB-231 and BT549 cells. PLE not only inhibited the viability of TNBC cells (Figures $4(\mathrm{a})$ and $4(\mathrm{~d})$ ) but also reduced their proliferation and migration (Figures 4(c), 4(f), and 5(d)). Examining the effect of PLE on Hippo-YAP signaling revealed that PLE inhibited the activation of YAP/ TAZ via phosphorylation (Figures 2(c), 5(a), and 5(b)). PLE also inhibited the binding between YAP and TEAD in HEK293A and MDA-MB-231 cells (Figures 2(d) and 5(c)). PLE downregulated the transcriptional activity of YAPTEAD (Figures 3(c) and 3(f)). In particular, the inhibitory effects of PLE on the expression of YAP-dependent genes were also abolished in LATS1/2 KO cells (Figure 3(c)). The cytotoxicity and apoptosis-inducing ability of PLE were also significantly attenuated in the LATS1/2 knockout clone (Figures S8(a) and S8(b)). These results suggest that PLE exhibits anticancer effects by inhibiting the activity of YAP through LATS-dependent YAP phosphorylation.

In this study, the anticancer efficacy of PLE through inhibition of YAP activity was clarified using an in vitro model. Hence, further studies are needed to verify the efficacy of PLE in animal models and to develop new therapeutic strategies using PLE to regulate the growth and proliferation of TNBCs.

\section{Abbreviations}

LATS1/2: Large tumor suppressor kinase $1 / 2$

PLE: $\quad$ Perilla frutescens var. acuta (Odash.) Kudo leaf extracts

TAZ: $\quad$ WW domain-containing transcription factor

TNBC: Triple-negative breast cancer

YAP: $\quad$ Yes-associated protein.

\section{Data Availability}

The data used in the study are available upon request to the corresponding author.

\section{Disclosure}

Cho-Long Kim and $\mathrm{Yu}-\mathrm{Su}$ Shin are the co-first authors.

\section{Conflicts of Interest}

The authors declare no conflicts of interest.

\section{Authors' Contributions}

C.L.K., Y.S.S., and H.S.J. designed, analyzed, and conducted experiments. S.H.C., S.R.O., and K.S.K. conducted experiments. C.L.K. assisted J.S.M. in writing the manuscript. J.S.M. and H.S.J. conceived the study and wrote the manuscript. All authors commented on the manuscript before submission. Cho-Long Kim and Yu-Su Shin equally contributed to this work.

\section{Acknowledgments}

This study was supported by the National Research Foundation of Korea (NRF) funded by the Ministry of Science, ICT and Future Planning (2016R1C1B2016135 and 2019R1F1A1060639) and the New Faculty Research Fund of Ajou University School of Medicine and by a grant from the 
Cooperative Research Program for Agriculture Science and Technology Development (project no. PJ01330501) to Y.S.S. and by the National Research Foundation of Korea (NRF) grant funded by the Korea Government (MISP) (2014R1A5A2009936 and 2018R1D1A1B07043856) to H.S.J.

\section{Supplementary Materials}

Figure S1. Perilla frutescens var. acuta (Odash.) Kudo leaf extract (PLE) induces YAP phosphorylation. PLE increased YAP phosphorylation. HEK293A cells were treated with various natural compounds $(50 \mu \mathrm{g} / \mathrm{mL})$ for $6 \mathrm{~h}$. Dimethyl sulfoxide (DMSO) was used as a control treatment. Phosphorylated proteins were detected using a phos-tag gel. Vinculin was used as the loading control. Figure S2. PLE has no effect on YAP mediated RUNX2-luferase reporter activity. HEK293A cells were co-transfected with a $6 \times$ OSE2luciferase reporter for RUNX2, with or without HA-YAP2. HEK293A cells were treated with PLE in a concentrationdependent manner. Cells were harvested $10 \mathrm{~h}$ after treatment. Representative results of a single experiment with $n=3$ biological replicates; three independent experiments were carried out. Luciferase activity was measured and normalized to that of co-transfected Renilla. Figure S3. PLE inhibited mRNA expression of YAP target genes in a MAP4K4/6/7-dependent manner. Wild-type and MAP4K4/ 6/7 KO HEK293A cells were treated with PLE for $12 \mathrm{~h}$, and then mRNA levels of CTGF and CYR61 were measured using RT-qPCR (error bars represent \pm SEM from $n=3$ per group). $* p<0.05, * * p<0.01$, and $* * * p<0.001$; Student's $t$-test (unpaired, one-tailed) was used for statistical analysis. Figure S4. Expression of YAP and TAZ on human $\mathrm{BC}$ and normal cell lines was analyzed by western blot. Cell lysates were subjected to immunoblotting with the indicated antibodies. Both YAP and TAZ were expressed predominantly in MDA-MB-231 and BT549 human BC cell lines. Figure S5. Effects of PLE on the viability of MCF10A cells. (a) MCF10A cells were treated using the indicated concentrations of PLE for $24 \mathrm{~h}$. Cell viability was measured by using the EZ-Cytox cell viability assay kit. IC50 values were calculated from concentration response curves using Prism 5.0 software. The IC50 value of MCF10A cells was $680.5 \mu \mathrm{g} / \mathrm{mL}$. Error bars represent \pm SEM from $n=3$ per group. (b) MCF10A cells were treated with DMSO and $100 \mu \mathrm{g} / \mathrm{mL}$ of PLE for 5 days. Cell proliferation was measured by SRB assay at an absorbance of $540 \mathrm{~nm}$. MCF10A cells with PLE were decreased by $14 \%$ compared with that of the control cells. Bars represent mean \pm SEM $(n=3) . * * * p<0.001$, $* * p<0.01$; Student's $t$-test (unpaired, one-tailed) was used for statistical analysis. Figure S6. PLE disrupts YAP-TEAD interaction in MDA-MB-231 cells. (a) Levels of TEAD expression among several $\mathrm{BC}$ cell lines. Gene expression analysis of the Broad-Novartis Cancer Cell Line Encyclopedia (CCLE) dataset showed that TEAD1-4 were highly expressed in various $\mathrm{BC}$ cell types. The $x$-axis indicates cancer cell types; $y$-axis indicates the target expression levels of TEAD1-4 in BC cell lines. (b) MDA-MB-231 cells were treated with PLE $(50 \mu \mathrm{g} / \mathrm{mL})$ for $6 \mathrm{~h}$. Endogenous YAP/TAZ was immunoprecipitated, and the co-precipitated TEAD was detected by western blot. Figure S7. TEAD1 $\triangle$ C-YAP (AD), a fusion of the TEAD DNA-binding domain with the YAP transactivation domain, was stably expressed in MDA-MB231 cells. Expression of HA-pPGS (empty vector) and HATEAD1 $\triangle \mathrm{C}$-YAP (AD) was confirmed using western blot, and the mRNA level of CYR61 was determined using RTqPCR (error bars represent \pm SEM from $n=3$ per group). Figure S8. LATS1/2 KO BT549 cells increased resistance to PLE inhibitory effects to a greater extent compared with wild-type cells. (a) BT549 LATS1/2 KO cells were treated with various concentrations $(0.001,0.01,0.1,1,10,100$, and $1000 \mu \mathrm{g} / \mathrm{mL}$ ) of PLE for $24 \mathrm{~h}$. Cell viability was measured by using the EZ-Cytox cell viability assay kit. IC50 values were determined from concentration response curves using Prism 5.0 Software. The IC50 value of BT549 LATS1/2 KO cells was $713 \mu \mathrm{g} / \mathrm{mL}$. Error bars represent \pm SEM from $n=3$ per group. (b) To measure levels of apoptosis-related protein, western blot was performed on wild-type and LATS1/2 KO BT549 cells treated with PLE at 50 and $100 \mu \mathrm{g} / \mathrm{mL}$ for $12 \mathrm{~h}$. The lysates were immunoblotted with the indicated antibodies. (Supplementary Materials)

\section{References}

[1] H. Yu, J. F. Qiu, L. J. Ma, Y. J. Hu, P. Li, and J. B. Wan, "Phytochemical and phytopharmacological review of Perilla frutescens L. (Labiatae), a traditional edible-medicinal herb in China," Food and Chemical Toxicology, vol. 108, pp. 375-391, 2017.

[2] X. J. Zhou, L. L. Yan, P. P. Yin et al., "Structural characterisation and antioxidant activity evaluation of phenolic compounds from cold-pressed Perilla frutescens var. arguta seed flour," Food Chemistry, vol. 164, pp. 150-157, 2014.

[3] M. Asif, "Phytochemical study of polyphenols in Perilla Frutescens as an antioxidant," Avicenna Journal of Phytomedicine, vol. 2, no. 4, pp. 169-178, 2012.

[4] H. J. Lim, K. W. Woo, K. R. Lee, S. K. Lee, and H. P. Kim, "Inhibition of proinflammatory cytokine generation in lung inflammation by the leaves of perilla frutescens and its constituents," Biomolecules Therapeutics (Seoul), vol. 22, no. 1, pp. 62-67, 2014.

[5] H. Ueda and M. Yamazaki, "Anti-inflammatory and antiallergic actions by oral administration of a perilla leaf extract in mice," Bioscience Biotechnology and Biochemistry, vol. 65, no. 7, pp. 1673-1675, 2001.

[6] T. Makino, Y. Furuta, H. Wakushima, H. Fujii, K. Saito, and Y. Kano, "Anti-allergic effect of Perilla frutescens and its active constituents," Phytotherapy Research, vol. 17, no. 3, pp. 240-243, 2003.

[7] Y. Kwak and J. Ju, "Inhibitory activities of Perilla frutescens britton leaf extract against the growth, migration, and adhesion of human cancer cells," Nutrition and Research Practice, vol. 9, no. 1, pp. 11-16, 2015.

[8] A. A. Abd El-Hafeez, T. Fujimura, R. Kamei et al., "Synergistic tumor suppression by a Perilla frutescens-derived methoxyflavanone and anti-cancer tyrosine kinase inhibitors in A549 human lung adenocarcinoma," Cytotechnology, vol. 70, no. 3, pp. 913-919, 2017.

[9] J. Cho, L. Tremmel, O. Rho et al., "Evaluation of pentacyclic triterpenes found in Perilla frutescens for inhibition of skin tumor promotion by 12-O-tetradecanoylphorbol-13-acetate," Oncotarget, vol. 6, no. 36, pp. 39292-39306, 2015. 
[10] H. Ueda, C. Yamazaki, and M. Yamazaki, "Luteolin as an antiinflammatory and anti-allergic constituent of Perilla frutescens," Biological and Pharmaceutical Bulletin, vol. 25, no. 9, pp. 1197-1202, 2002.

[11] N. Banno, T. Akihisa, H. Tokuda et al., "Triterpene acids from the leaves of Perilla frutescens and their anti-inflammatory and antitumor-promoting effects," Bioscience Biotechnology and Biochemistry, vol. 68, no. 1, pp. 85-90, 2004.

[12] J. Dong, G. Feldmann, J. Huang et al., "Elucidation of a universal size-control mechanism in Drosophila and mammals," Cell, vol. 130, no. 6, pp. 1120-1133, 2007.

[13] S. W. Plouffe, A. W. Hong, and K. L. Guan, "Disease implications of the Hippo/YAP pathway," Trends in Molecular Medicine, vol. 21, no. 4, pp. 212-222, 2015.

[14] F. X. Yu and K. L. Guan, "The Hippo pathway: regulators and regulations," Genes and Development, vol. 27, no. 4, pp. 355-371, 2013.

[15] C. G. Hansen, T. Moroishi, and K. L. Guan, "YAP and TAZ: a nexus for Hippo signaling and beyond," Trends Cell Biology, vol. 25, no. 9, pp. 499-513, 2015.

[16] B. Zhao, X. Wei, W. Li et al., "Inactivation of YAP oncoprotein by the Hippo pathway is involved in cell contact inhibition and tissue growth control," Genes and Development, vol. 21, no. 21, pp. 2747-2761, 2007.

[17] M. Overholtzer, J. Zhang, G. A. Smolen et al., “Transforming properties of YAP, a candidate oncogene on the chromosome 11q22 amplicon," Proceedings of the National Academy of Sciences of the United States of America, vol. 103, no. 33, pp. 12405-12410, 2006.

[18] I. Vaz-Luis, R. A. Ottesen, M. E. Hughes et al., "Outcomes by tumor subtype and treatment pattern in women with small, node-negative breast cancer: a multi-institutional study," Journal of Clinical Oncology, vol. 32, no. 20, pp. 2142-2150, 2014.

[19] S. P. Shah, A. Roth, R. Goya et al., "The clonal and mutational evolution spectrum of primary triple-negative breast cancers," Nature, vol. 486, no. 7403, pp. 395-399, 2012.

[20] J. Collignon, L. Lousberg, H. Schroeder, and G. Jerusalem, "Triple-negative breast cancer: treatment challenges and solutions," Breast Cancer (Dove Med Press), vol. 8, pp. 93-107, 2016.

[21] J. Zhang, S. Yao, Q. Hu et al., "Genetic variations in the Hippo signaling pathway and breast cancer risk in African American women in the AMBER consortium," Carcinogenesis, vol. 37, no. 10, pp. 951-956, 2016.

[22] S. S. Chang, H. Yamaguchi, W. Xia et al., "Aurora A kinase activates YAP signaling in triple-negative breast cancer," Oncogene, vol. 36, no. 9, pp. 1265-1275, 2017.

[23] L. Guo, J. Zheng, J. Zhang, H. Wang, G. Shao, and L. Teng, "Knockdown of TAZ modifies triple-negative breast cancer cell sensitivity to EGFR inhibitors by regulating YAP expression," Oncology Reports, vol. 36, no. 2, pp. 729-736, 2016.

[24] L. Wu and X. Yang, "Targeting the Hippo pathway for breast cancer therapy," Cancers (Basel), vol. 10, 11 pages, 2018.

[25] J. S. Mo, "The role of extracellular biophysical cues in modulating the Hippo-YAP pathway," BMB Reports, vol. 50, no. 2, pp. 71-78, 2017.

[26] Z. Meng, T. Moroishi, V. Mottier-Pavie et al., "MAP4K family kinases act in parallel to MST1/2 to activate LATS1/2 in the Hippo pathway," Nature Communications, vol. 6, p. 8357, 2015.

[27] J. S. Mo, Z. Meng, Y. C. Kim et al., "Cellular energy stress induces AMPK-mediated regulation of YAP and the Hippo pathway," Nature Cell Biology, vol. 17, no. 4, pp. 500-510, 2015.

[28] Y. Peng, J. Ye, and J. Kong, "Determination of phenolic compounds in Perilla frutescens L. by capillary electrophoresis with electrochemical detection," Journal of Agricultural Food Chemistry, vol. 53, no. 21, pp. 8141-8147, 2005.

[29] B. Zhao, L. Li, K. Tumaneng, C. Y. Wang, and K. L. Guan, "A coordinated phosphorylation by Lats and CK1 regulates YAP stability through SCF (beta-TRCP)," Genes and Development, vol. 24, no. 1, pp. 72-85, 2010.

[30] B. Zhao, Q. Y. Lei, and K. L. Guan, "The Hippo-YAP pathway: new connections between regulation of organ size and cancer," Current Opinion in Cell Biology, vol. 20, no. 6, pp. 638-646, 2008.

[31] R. Yagi, L. F. Chen, K. Shigesada, Y. Murakami, and Y. Ito, "A WW domain-containing yes-associated protein (YAP) is a novel transcriptional co-activator," EMBO Journal, vol. 18, no. 9, pp. 2551-2562, 1999.

[32] C. L. Kim, S. H. Choi, and J. S. Mo, "Role of the Hippo pathway in fibrosis and cancer," Cells, vol. 8 , no. 5, p. 468, 2019.

[33] B. Zhao, X. Ye, J. Yu et al., "TEAD mediates YAP-dependent gene induction and growth control," Genes and Development, vol. 22, no. 14, pp. 1962-1971, 2008.

[34] F. X. Yu, B. Zhao, and K. L. Guan, "Hippo pathway in organ size control, tissue homeostasis, and cancer," Cell, vol. 163, no. 4, pp. 811-828, 2015.

[35] E. H. Chan, M. Nousiainen, R. B. Chalamalasetty, A. Schafer, E. A. Nigg, and H. H. Sillje, "The Ste20-like kinase Mst2 activates the human large tumor suppressor kinase Lats1," Oncogene, vol. 24, no. 12, pp. 2076-2086, 2005.

[36] H. Glantschnig, G. A. Rodan, and A. A. Reszka, "Mapping of MST1 kinase sites of phosphorylation. Activation and autophosphorylation," Journal of Biological Chemistry, vol. 277, no. 45, pp. 42987-42996, 2002.

[37] M. Praskova, A. Khoklatchev, S. Ortiz-Vega, and J. Avruch, "Regulation of the MST1 kinase by autophosphorylation, by the growth inhibitory proteins, RASSF1 and NORE1, and by Ras," Biochemical Journal, vol. 381, no. Pt 2, pp. 453-462, 2004.

[38] Y. Zheng, W. Wang, B. Liu, H. Deng, E. Uster, and D. Pan, "Identification of happyhour/MAP4K as alternative HPO/ MST-like kinases in the Hippo kinase cascade," Development Cell, vol. 34, no. 6, pp. 642-655, 2015.

[39] S. Li, Y. S. Cho, T. Yue, Y. T. Ip, and J. Jiang, "Overlapping functions of the MAP4K family kinases Hppy and Msn in Hippo signaling," Cell Discovery, vol. 1, Article ID 15038, 2015.

[40] K. F. Harvey, X. Zhang, and D. M. Thomas, "The Hippo pathway and human cancer," Nature Reviews Cancer, vol. 13, no. 4, pp. 246-257, 2013.

[41] J. S. Mo, H. W. Park, and K. L. Guan, "The Hippo signaling pathway in stem cell biology and cancer," EMBO Reports, vol. 15, no. 6, pp. 642-656, 2014.

[42] K. Zhang, H. X. Qi, Z. M. Hu et al., "YAP and TAZ take center stage in cancer," Biochemistry, vol. 54, no. 43, pp. 6555-6566, 2015.

[43] K. J. Kaneko, E. B. Cullinan, K. E. Latham, and M. L. DePamphilis, "Transcription factor mTEAD-2 is selectively expressed at the beginning of zygotic gene expression in the mouse," Development, vol. 124, no. 10, pp. 1963-1973, 1997.

[44] A. Vassilev, K. J. Kaneko, H. Shu, Y. Zhao, and M. L. DePamphilis, "TEAD/TEF transcription factors utilize the activation domain of YAP65, a SRC/Yes-associated 
protein localized in the cytoplasm," Genes and Development, vol. 15, no. 10, pp. 1229-1241, 2001.

[45] C. E. Yockey, G. Smith, S. Izumo, and N. Shimizu, "cDNA cloning and characterization of murine transcriptional enhancer factor-1-related protein 1, a transcription factor that binds to the M-CAT motif," Journal of Biological Chemistry, vol. 271, no. 7, pp. 3727-3736, 1996.

[46] S. W. Chan, C. J. Lim, K. Guo et al., "A role for TAZ in migration, invasion, and tumorigenesis of breast cancer cells," Cancer Research, vol. 68, no. 8, pp. 2592-2598, 2008.

[47] J. M. Lamar, P. Stern, H. Liu, J. W. Schindler, Z. G. Jiang, and R. O. Hynes, "The Hippo pathway target, YAP, promotes metastasis through its TEAD-interaction domain," Proceedings of the National Academy of Sciences of the United States of America, vol. 109, no. 37, pp. E2441-E2450, 2012.

[48] L. A. Torre, F. Bray, R. L. Siegel, J. Ferlay, J. Lortet-Tieulent, and A. Jemal, "Global cancer statistics, 2012," CA A Cancer Journal for Clinicians, vol. 65, no. 2, pp. 87-108, 2015.

[49] S. Cleator, W. Heller, and R. C. Coombes, "Triple-negative breast cancer: therapeutic options," Lancet Oncology, vol. 8, no. 3, pp. 235-244, 2007.

[50] R. Dent, M. Trudeau, K. I. Pritchard et al., "Triple-negative breast cancer: clinical features and patterns of recurrence," Clinical Cancer Research, vol. 13, no. 15 Pt 1, pp. 4429-4434, 2007.

[51] F. D. Camargo, S. Gokhale, J. B. Johnnidis et al., "YAP1 increases organ size and expands undifferentiated progenitor cells," Current Biology, vol. 17, no. 23, pp. 2054-2060, 2007.

[52] R. Johnson and G. Halder, "The two faces of Hippo: targeting the Hippo pathway for regenerative medicine and cancer treatment," Nature Reviews Drug Discovery, vol. 13, no. 1, pp. 63-79, 2014.

[53] D. Yimlamai, C. Christodoulou, G. G. Galli et al., "Hippo pathway activity influences liver cell fate," Cell, vol. 157, no. 6, pp. 1324-1338, 2014.

[54] M. Cordenonsi, F. Zanconato, L. Azzolin et al., "The Hippo transducer TAZ confers cancer stem cell-related traits on breast cancer cells," Cell, vol. 147, no. 4, pp. 759-772, 2011.

[55] J. Diaz-Martin, M. A. Lopez-Garcia, L. Romero-Perez et al., "Nuclear TAZ expression associates with the triple-negative phenotype in breast cancer," Endocrine Related Cancer, vol. 22, no. 3, pp. 443-454, 2015.

[56] Y. W. Li, J. Xu, G. Y. Zhu et al., "Apigenin suppresses the stem cell-like properties of triple-negative breast cancer cells by inhibiting YAP/TAZ activity," Cell Death Discovery, vol. 4, p. 105, 2018.

[57] H. Yuan, Q. Ma, L. Ye, and G. Piao, "The traditional medicine and modern medicine from natural products," Molecules, vol. 21, no. 5, p. 559, 2016.

[58] H. F. Ji, X. J. Li, and H. Y. Zhang, "Natural products and drug discovery. can thousands of years of ancient medical knowledge lead us to new and powerful drug combinations in the fight against cancer and dementia?" EMBO Reports, vol. 10, no. 3, pp. 194-200, 2009.

[59] Y. Lin, R. Shi, X. Wang, and H. M. Shen, "Luteolin, a flavonoid with potential for cancer prevention and therapy," Current Cancer Drug Targets, vol. 8, no. 7, pp. 634-646, 2008.

[60] D. Y. Lim, Y. Jeong, A. L. Tyner, and J. H. Park, "Induction of cell cycle arrest and apoptosis in HT-29 human colon cancer cells by the dietary compound luteolin," American Journal of Physiology-Gastrointestinal and Liver Physiology, vol. 292, no. 1, pp. G66-G75, 2007.

[61] K. Selvendiran, H. Koga, T. Ueno et al., "Luteolin promotes degradation in signal transducer and activator of transcription
3 in human hepatoma cells: an implication for the antitumor potential of flavonoids," Cancer Research, vol. 66, no. 9, pp. 4826-4834, 2006.

[62] N. Osakabe, A. Yasuda, M. Natsume, and T. Yoshikawa, "Rosmarinic acid inhibits epidermal inflammatory responses: anticarcinogenic effect of Perilla frutescens extract in the murine two-stage skin model," Carcinogenesis, vol. 25, no. 4, pp. 549-557, 2004.

[63] Y. G. Hur, Y. Yun, and J. Won, "Rosmarinic acid induces p56lck-dependent apoptosis in Jurkat and peripheral T cells via mitochondrial pathway independent from FAS/FAS ligand interaction," Journal of Immunology, vol. 172, no. 1, pp. 79-87, 2004.

[64] D. O. Moon, M. O. Kim, J. D. Lee, Y. H. Choi, and G. Y. Kim, "Rosmarinic acid sensitizes cell death through suppression of TNF-alpha-induced NF-kappaB activation and ROS generation in human leukemia U937 cells," Cancer Letters, vol. 288, no. 2, pp. 183-191, 2010. 